\title{
Efeitos do tabagismo na funcionalidade pulmonar
}

\section{Tobaccoism effects on pulmonary function}

\author{
Kelser de Souza Kock ${ }^{1}$, Hektor França Barros ${ }^{1}$ \\ 1. Universidade do Sul de Santa Catarina (UNISUL), Santa Catarina, Brasil.
}

\begin{abstract}
Resumo
O tabagismo é um hábito frequente entre os brasileiros; ele é causador de doenças que podem ser evitadas, e um dos maiores causadores de morte por câncer de pulmão, doença coronariana, DPOC e AVC. 0 objetivo deste estudo foi avaliar a funcionalidade pulmonar, a dispneia, os índices socioeconômicos e o perfil dos pacientes relacionados com o tabagismo, em pacientes que participam do programa de controle do tabagismo do SUS em Tubarão - SC no ano de 2013. Foram avaliados 39 pacientes, sendo 76,9\% mulheres, com idade média de $52 \pm 10$ anos e IMC médio de $28 \pm 4 \mathrm{~kg} / \mathrm{m} 2$. A carga tabágica foi de 44 anosmaço, com $41 \%$ dos indivíduos com dependência grave à nicotina, HAS e depressão como morbidades mais frequentes, com escolaridade principalmente o ensino fundamental incompleto (46,2\%) e 51,2\% com renda de até um salário mínimo. Foram demonstradas associações significativas entre tabagismo (anos-maço) e dependência à nicotina $(r=0,494 ; p=0,001)$, dispneia $(r=0,424 ; p=0,007)$ e variáveis espirométricas: VEF1 $(r=-0,323 ; p=0,045)$, VEF1) CVF $(r=-0,391 ; p=0,014)$ e FEF25-75 ( $r=-0,338 ; p=0,035)$. A função pulmonar média dos pacientes foi considerada normal. As variáveis relacionadas à obstrução pulmonar estão associadas à carga de tabagismo, destacando-se o papel da espirometria como ferramenta para a identificação dos tabagistas com probabilidade de desenvolver doença respiratória.
\end{abstract}

Palavras-chave: Hábito de fumar. Espirometria. Dispneia. Doença Pulmonar Obstrutiva Crônica.

\begin{abstract}
Tobaccoism is as frequent habit among Brazilians, which causes diseases that may be preventable and one of the leading causes of death from lung cancer, coronary disease, COPD and stroke. The aim of this study was to evaluate the pulmonary functionality, dyspnea, socioeconomic rates and the profile of the patients related with smoking, in patients who participate of the tobacco control program of SUS in Tubarão, in the state of Santa Catarina. Thirty nine patients have been evaluated of whom 76,9\% were women, with an average age of $52 \pm 10$ years and average BMI $28 \pm 4 \mathrm{~kg} / \mathrm{m} 2$. The tobacco intake was 44 pack-years, with $41 \%$ of individuals with severe nicotine dependence, hypertension and depression as the most frequent morbidities; mainly incomplete elementary school education $(46,2 \%)$ and $51,2 \%$ with wage income up a minimum wage. It has been demonstrated significant associations between smoking (pack-years) and nicotine dependence $(r=0,494, p=0,001)$, dyspnea $(r=0,424, p=0,007)$ and spirometric variables: FEV1 $(r=-0,323$, $p=0,045)$, FEV1/FVC $(r=-0,391, p=0,014)$ and FEF25-75 $(r=-0,338, p=0,035)$. The average pulmonary function of patients was considered normal. It is concluded that the variables related to pulmonary obstruction are associated with the amount of tobaccoism, emphasizing the role of spirometry as a tool for identification of smokers that are likely to develop respiratory illness.
\end{abstract}

Keywords: Habit of smoking. Spirometry. Dyspnea. Chronic Obstructive Pulmonary Disease.

\section{INTRODUÇÃO}

Durante séculos, o uso do tabaco difundiu-se nas Américas, mas apenas no final do século XIX e, sobretudo, na primeira metade do século $X X$ que a produção de cigarros em escala industrial e o processo de propaganda e marketing difundiram seu uso social ${ }^{1}$.

O Tabagismo é considerado a segunda causa de morte evitável no mundo, de acordo com a Organização Mundial da Saúde. Entre os desfechos relacionados à utilização do tabaco, podem ser descritos diversos tipos de câncer (pulmão, boca, laringe esôfago, estômago, pâncreas, bexiga, rim, colo do útero e leucemia mieloide aguda), DPOC, doença coronariana, hipertensão arterial e acidente vascular encefálico. O simples fato de estar exposto à fumaça de produtos de tabaco contribui para o desenvolvimento ou para o agravamento de diversos outros males ${ }^{2}$.

Na revisão sistemática realizada por Oliveira, Valente e Leite ${ }^{3}$, a qual relaciona aspectos da mortalidade atribuída ao tabaco, considerou-se que as mortes relacionadas ao tabagismo são mais frequentes em homens que em mulheres e entre as doenças relacionadas ao tabagismo estão: câncer de traqueia, brônquio e pulmão, doenças isquêmicas cardíacas, DPOC e doenças cérebro-vasculares.

Calcula-se que o fumo seja responsável por mais de cinco milhões de morte a cada ano. Quanto ao fumo passivo, estimase que provoque cerca de 600 mil mortes anuais de indivíduos expostos à fumaça do tabaco ${ }^{2}$. A dependência nicotínica parece ser a causa da persistência do tabagismo e da dificuldade de sua suspensão. O grau de dependência de nicotina pode ser estimado pelo Questionário de Tolerância de Fagerström, que tem sido mundialmente utilizado como ferramenta de avaliação desta dependência em substituição a testes que envolvem altos custos financeiros, exige mais tempo ou são invasivos ${ }^{4}$.

Mesmo em indivíduos com baixo grau de dependência e de consumo de cigarros, os sintomas respiratórios, como tosse

Correspondência: Kelser de Souza Kock. Endereço: Rua Galdino José de Bessa, 164, Oficinas, Tubarão - SC. CEP: 88702-220. Telefone: (48)9996-9811. E-mail: kelserkock@yahoo.com.br ou kelser.kock@unisul.br

Conflito de interesse: Não há conflito de interesse por parte de qualquer um dos autores.

Recebido em: 23 Set 2014; Revisado em: 28 Out 2014; Aceito em: 18 Nov 2014. 
e falta de ar estão presentes. Quanto maior a dependência, maior a gravidade desses sintomas. Conforme alguns pesquisadores, a espirometria pode identificar tabagistas com risco de desenvolver doença respiratória. Comentam ainda, a importância da manutenção de programas de controle do tabagismo em ambiente universitário ${ }^{5}$.

A espirometria é um exame que avalia fluxos e volumes pulmonares e é indicada para a avaliação de sintomas respiratórios, classificação funcional de pneumopatias, análise da resposta terapêutica e acompanhamento de pacientes crônicos portadores de doenças respiratórias ${ }^{6}$. Outro exame ventilatório importante é a manovacuometria, que monitora as pressões inspiratórias e expiratórias máximas (PImáx e PEmáx). Essas pressões avaliam de forma indireta o grau de força muscular respiratória. Quando os valores de PImáx e PEmáx estiverem abaixo do previsto, é constatada fraqueza muscular respiratória7.

O tabagismo tornou-se o maior causador de doenças evitáveis no mundo, entre essas, a DPOC que inclui enfisema pulmonar e bronquite crônica; a fumaça inalada leva a uma reposta inflamatória todas as vezes que é inalada; por isso, a interrupção resulta em melhoras funcionais e estruturais da arquitetura pulmonar $^{8}$.

A cessação do tabagismo traz consideráveis benefícios à saúde, pois indivíduos que param de fumar evitam a maioria das doenças associadas ao tabaco. Apesar de todo o conhecimento científico acumulado sobre os riscos do tabaco, as tendências do seu consumo ainda são alarmantes. Um terço da população mundial, com 15 anos ou mais, é fumante. No Brasil, 9\% da população são dependentes de tabaco, sendo que $16,4 \%$ desejam parar ou reduzir seu consumo².

No Brasil, a faixa etária para a iniciação do consumo de produtos do tabaco começa entre 17 a $19 \operatorname{anos}^{1}$. A proporção de mulheres que começam a fumar antes dos 15 anos é superior à dos homens. Um fator que impacta a idade de iniciação é o nível de instrução, sendo elevada a proporção dos indivíduos sem instrução ou com menos de um ano de estudo. A renda de indivíduos e sua associação com a idade de iniciação apresenta padrão similar. Em 2008, 17,5\% da população brasileira com 15 anos ou mais eram usuários de algum tipo de tabaco. 0 percentual de fumantes é maior nas áreas rurais do que nas urbanas $^{2}$.

Nesse sentido, a avaliação dos efeitos do tabagismo na funcionalidade pulmonar é importante, pois pode identificar problemas respiratórios, além de auxiliar futuras estratégias para controle do tabagismo. Assim, o objetivo deste trabalho foi avaliar os efeitos do tabagismo na funcionalidade pulmonar em indivíduos inseridos no programa de controle do tabagismo do Sistema Único de Saúde (SUS) em Tubarão - SC.

\section{MÉTODOS}

Estudo transversal, descritivo. População: indivíduos inseridos no programa de controle do tabagismo do SUS em Tubarão - SC no ano de 2013. A amostragem foi definida de forma intencional não probabilística, pelas Unidades Básicas de Saúde (UBS) que iniciaram o programa em 2013 e por aqueles que aceitarem participar da pesquisa e assinar o Termo de Consentimento Livre e Esclarecido. Foram excluídos os pacientes que, após mais de três tentativas de ligação telefônica não foram contactados, ou faltaram à avaliação.

Para a coleta de dados foi realizado um contato prévio com a Secretária da Saúde de Tubarão - SC, para a do projeto e formalização da pesquisa. Por meio de contato telefônico, os pacientes cadastrados no programa de controle de tabagismo do município foram convidados para a participação do trabalho. Os participantes foram encaminhados à Clínica Escola de Fisioterapia da Universidade do Sul de Santa Catarina (UNISUL), Campus Tubarão - SC, sendo submetidos à anamnese e à realização dos testes ventilatórios.

$\mathrm{Na}$ anamnese, foram coletadas idade e gênero, variáveis antropométricas de peso e altura, para a do índice de massa corpórea (IMC) por meio da equação IMC=peso/altura ${ }^{2}$, dados socioeconômicos relacionados à renda, à escolaridade e à profissão, ao índice de tabagismo anos maço $=$ [(número médio de cigarros fumados ao dia $\div 20$ ) * número de anos de tabagismo $]^{8}$, nível de dispneia conforme escala do Medical Research Council (MRC) ${ }^{8}$ e dependência à nicotina pelo teste de Fagerstrom ${ }^{4}$.

Os testes ventilatórios realizados foram a espirometria para a medida da função pulmonar, sendo coletadas as variáveis CVF, VEF1 e VEF1/CVF; e a manovacuometria, para medida das pressões respiratórias, coletando os dados de PImáx e PEmáx. Os mesmos seguiram as diretrizes para testes de função pulmonar 6 .

Os dados foram armazenados em um banco de dados criado com o auxílio do software Excel ${ }^{\circledR}$, posteriormente exportado para o software SPSS $20.0^{\circledR}$. Os dados foram demonstrados por meio de números absolutos e percentuais, medidas de tendência central e dispersão. A análise foi realizada por meio da correlação de Pearson $(p<0,05)$ pelo teste $U$ de Man-Whitney $(p<0,05)$.

O projeto de pesquisa foi submetido ao Comitê de Ética e Pesquisa em Seres Humanos da UNISUL, aprovado sob o número 414.579.

\section{RESULTADOS E DISCUSSÃO}

Sessenta e cinco pacientes foram inseridos no programa de controle ao tabagismo do SUS em Tubarão - SC, destes, compuseram a amostra do estudo 39 (60\%) indivíduos, de acordo com os critérios prévios de elegibilidade. O programa de controle do tabagismo foi estabelecido nas seguintes UBS: Campestre, Recife, Santo Antônio e Serviço de Atendimento Integrado à Saúde (SAIS). As demais UBS não iniciaram o 
programa de controle ao tabagismo no ano de 2013.

As características gerais do grupo em estudo demonstrou uma faixa etária média compatível com os adultos de meia idade, que apresentavam sobrepeso, segundo o IMC. Observa-se que a maior parte da população estudada é composta por mulheres $(76,9 \%)$, trabalhadoras do Lar $(20,5 \%)$ ou aposentadas $(25,7 \%)$. Com relação ao grau de escolaridade, a maioria foi composta por pessoas que cursaram o ensino fundamental incompleto (46,2\%), com renda de um salário mínimo (51,3\%) e apresentando Hipertensão Arterial sistêmica como doença mais prevalente $(30,8 \%)$ seguida de Transtorno Depressivo $(23,1 \%)$ (Tabela 1$)$.

Tabela 1. Características gerais dos pacientes do Programa de controle do tabagismo- Tubarão, 2013.

\begin{tabular}{|c|c|}
\hline Variáveis & $n(\%)$ \\
\hline Idade (anos) ${ }^{*}$ & $52( \pm 10)$ \\
\hline $\mathrm{IMC}\left(\mathrm{kg} / \mathrm{m}^{2}\right)^{*}$ & $28( \pm 4)$ \\
\hline \multicolumn{2}{|l|}{ Gênero } \\
\hline Masculino & $9(23,1)$ \\
\hline Feminino & $30(76,9)$ \\
\hline \multicolumn{2}{|l|}{ Escolaridade } \\
\hline Fundamental incompleto & $18(46,2)$ \\
\hline Fundamental & $10(25,6)$ \\
\hline Médio & $7(17,9)$ \\
\hline Superior & $1(2,6)$ \\
\hline Pós-graduação & $3(7,7)$ \\
\hline \multicolumn{2}{|l|}{ Renda } \\
\hline até 1 salário & $20(51,3)$ \\
\hline 1 a 3 salários & $11(28,2)$ \\
\hline mais que 3 & $18(20,5)$ \\
\hline \multicolumn{2}{|l|}{ Profissão } \\
\hline Do Lar & $10(25,7)$ \\
\hline Serviços Gerais & $6(15,4)$ \\
\hline Comércio & $5(12,8)$ \\
\hline Educação & $3(7,7)$ \\
\hline Saúde & $3(7,7)$ \\
\hline Costura & $3(7,7)$ \\
\hline Cozinha & $3(7,7)$ \\
\hline Mecânica & $3(7,7)$ \\
\hline Outros & $3(7,7)$ \\
\hline \multicolumn{2}{|l|}{ Morbidades } \\
\hline Hipertensão arterial sistemica & $12(30,8$ \\
\hline Depressão & $9(23,1)$ \\
\hline Doença osteo-musculares & $6(15,4)$ \\
\hline Diabetes mellitus & $5(12,8)$ \\
\hline Asma & $5(12,8)$ \\
\hline Acidente vascular cerebral & $2(5,1)$ \\
\hline Outras ${ }^{* *}$ & $6(15,4)$ \\
\hline
\end{tabular}

Em relação ao tabagismo e à dispneia, foi demonstrado um índice de dependência à nicotina muito elevado tendendo para o grau mais grave. Os pacientes tiveram uma elevada taxa de anos-maço fumados; porém, quando é analisada a presença de dispneia, verifica-se que apenas dois indivíduos apresentaram ao repouso (grau 4), sendo a maioria sintomática somente durante a atividade física. (Tabela 2)

Tabela 2. Características relacionadas ao tabagismo e dispneias dos pacientes do Programa de controle do tabagismo- Tubarão, 2013.

\begin{tabular}{lr}
\hline Variáveis & Resultados \\
\hline Tabagismo (anos.maço) & $44( \pm 26)$ \\
Média ( \pm desvio padrão) & \\
Dependência à nicotina (Fagerström) & $5,9( \pm 2,4)$ \\
Média ( \pm desvio padrão) & \\
Classificação - n(\%) & $11(28,2)$ \\
Leve & $12(30,8)$ \\
Moderado & $16(41,0)$ \\
Grave & \\
Dispneia & $1,2( \pm 1,3)$ \\
Média \pm desvio padrão & \\
Classificação - n(\%) & $18(46,2)$ \\
0 & $7(17,9)$ \\
1 & $5(12,8)$ \\
2 & $7(17,9)$ \\
3 & $2(5,1)$ \\
4 &
\end{tabular}

A tabela 3 apresenta as variáveis relacionadas à funcionalidade pulmonar. Pode-se observar que, considerando o limite da normalidade como $80 \%$ do valor previsto ${ }^{6}$, os resultados apresentaram-se em média sem alterações.

Tabela 3. Funcionalidade pulmonar

\begin{tabular}{lr}
\hline Variáveis & Média ( \pm desvio padrão) \\
\hline CVF (\%) & $85( \pm 13)$ \\
VEF1 (\%) & $82( \pm 16)$ \\
VEF1/CVF (\%) & $78( \pm 10)$ \\
FEF25-75 (\%) & $84( \pm 36)$ \\
PImáx (\%) & $94( \pm 34)$ \\
PEmáx (\%) & $118( \pm 39)$
\end{tabular}

CVF: Capacidade Vital Forçada, VEF1: Volume expiratório final no primeiro segundo, FEF25-75: Fluxo expiratório forçado entre 25 e 75\% da CVF, PImáx: Pressão inspiratória máxima, PEmáx: Pressão expiratória máxima

A Figura 1 apresenta os gráficos que correlacionam o tabagismo (anos-maço) com sintoma de dispneia, dependência à nicotina e variáveis da espirometria. Foram demonstradas associações significativas entre tabagismo (anos-maço) e dependência à nicotina, dispneia e variáveis espirométricas.

hipotireoidismo, artrose, osteoporose. 
Figura 1. Gráficos de correlação entre tabagismo (anos/maço), sintomas de dispneia, dependência à nicotina e variáveis espirométricas dos pacientes do Programa de controle do tabagismo-Tubarão
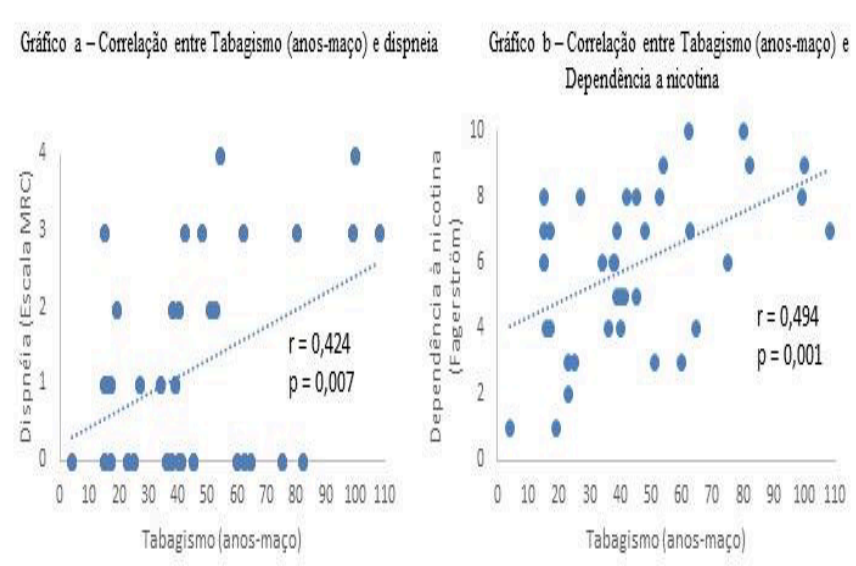
Dependència a nicotina

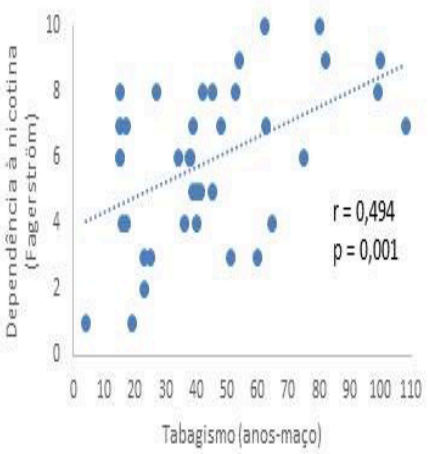

Gráfico c - Correlaçào entre Tabagismo (anos-maço) e VEF1 Giáfico d-Correlaçào entre Tabagismo (anos-maço) e VEF1/CVF
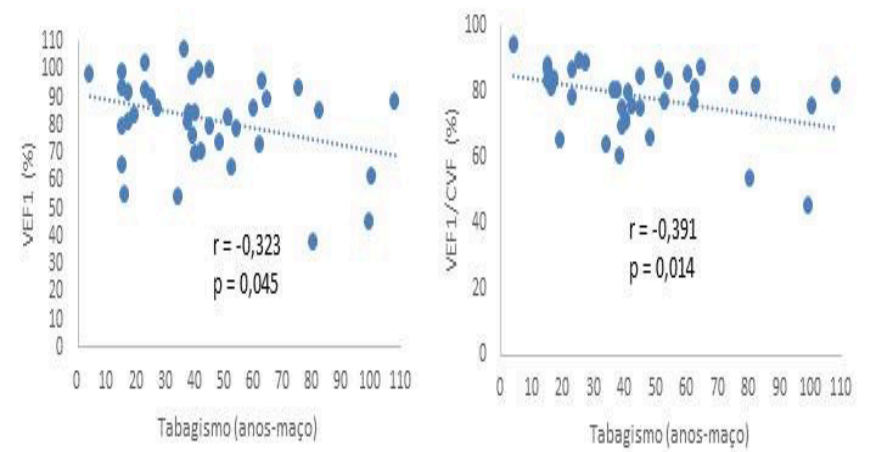

Gráfico e - Correlaçào entre Tabagismo (anos-maço) e FEF $25-75$

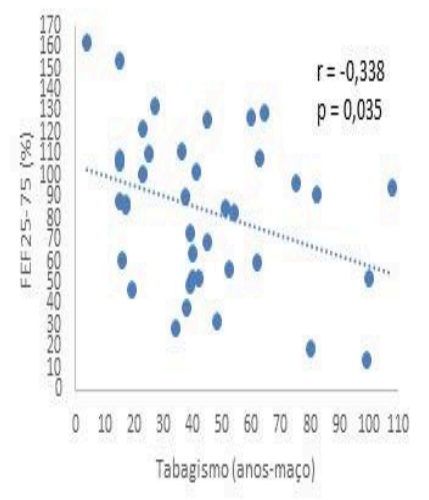

Ao comparar variáveis espirométricas e tabagismo (anos/maço) com dispneia, foi considerada a pontuação 0 e 1 como ausência de dispneia e pontuação 2, 3 e 4 como presença de dispneia. Foi observada diferença significativa no VEF1, VF1/CVF, FEF e no tabagismo (anos-maço), enquanto as variáveis CVF, PImax e PEmax não obtiveram diferença estatística (Tabela 4).
Tabela 4. Comparação entre função pulmonar, tabagismo e dispneia nos pacientes do Programa de controle do tabagismo - Tubarão, 2013.

\begin{tabular}{|c|c|c|c|}
\hline \multirow[b]{2}{*}{ Variáveis } & \multicolumn{2}{|c|}{$\begin{array}{l}\text { Dispneia } \\
\text { Media ( } \pm \text { desvio padrão) }\end{array}$} & \multirow[b]{2}{*}{$\mathbf{P}$} \\
\hline & Não & Sim & \\
\hline CVF (\%) & $86( \pm 13)$ & $84( \pm 15)$ & 0,534 \\
\hline VEF1 (\%) & $87( \pm 14)$ & $73( \pm 16)$ & 0,008 \\
\hline VEF1/CVF (\%) & $82( \pm 7)$ & $72( \pm 12)$ & 0,010 \\
\hline FEF25-75 (\%) & $100( \pm 32)$ & $57( \pm 25)$ & $<0,001$ \\
\hline PImáx (\%) & $100( \pm 34)$ & $82( \pm 31)$ & 0,076 \\
\hline PEmáx (\%) & $103( \pm 26)$ & $126( \pm 43)$ & 0,071 \\
\hline Tabagismo (anos-maço) & $36( \pm 21)$ & $58( \pm 29)$ & 0,014 \\
\hline
\end{tabular}

\section{DISCUSSÃO}

Os resultados obtidos relacionados às características gerais da amostra foram, principalmente, indivíduos na maior parte do gênero feminino, com leve sobrepeso, de meia idade, pouca escolaridade, baixa renda, ocupação principal: serviços gerais, dona de casa e aposentados, com comorbidades mais comuns a HAS e a depressão. Esses resultados estão de acordo com outros estudos, segundo dados do Ministério da $S a^{\prime} d^{2}$. Muitas pessoas que procuram parar com o tabagismo são do gênero feminino; mais da metade dos ex-tabagistas são mulheres, à medida que cresce o grau de escolaridade, diminui o percentual de fumantes. A classe econômica menos privilegiada economicamente tem uma maior prevalência de tabagistas ${ }^{2}$. Segundo um estudo de Godoy et al. ${ }^{9}$ em um programa de cessação ao tabagismo utilizando a espirometria como ferramenta para o diagnóstico de DPOC, encontrou que a maioria das pessoas era do sexo feminino de meia idade e um terço dos pacientes preencheram critérios de diagnóstico para DPOC.

É importante salientar que, por motivos socioculturais, a busca e o cuidado pela saúde ocorrem principalmente entre as mulheres ${ }^{10}$. Por esse motivo, a maior parte da amostra do presente estudo foi composta por esse gênero. No entanto, de forma geral, a maior prevalência de tabagismo dá-se entre os homens. Em 2010, a prevalência média de fumantes ativos era de $16,1 \%$. A prevalência foi maior nos indivíduos do sexo masculino $(20,5 \%)$ quando comparados aos do sexo feminino $(12,4 \%)$ e foi particularmente alta entre aqueles com até oito anos de escolaridade ${ }^{11}$. Cardoso, et al. ${ }^{12}$ avaliaram 494 funcionários de uma repartição pública da procuradoria geral da república mostrou que $11,7 \%$ dos entrevistados eram fumantes contra uma média de $18 \%$ de fumantes no Brasil ${ }^{2}$. A diferença na prevalência de tabagismo observado está relacionada ao fato de que $70 \%$ da população estudada possuíam ensino superior completo, além disso, pessoas religiosas e que praticavam atividade física fumavam menos.

Os níveis de tabagismo e dependência a nicotina nesse estudo foram considerada altos. Esse dado é corroborado por outras pesquisas $^{13,14}$, demonstrando que em programas de controle ao tabagismo, a maior parte dos indivíduos possui esse perfil. 
Em contrapartida, em um estudo realizado com 629 pacientes no Hospital de Clínicas da Universidade Federal do Paraná, em Curitiba, foi identificada a presença de $12,1 \%$ fumantes, $28,5 \%$ ex-fumantes e $59,5 \%$ não fumantes. A carga tabágica entre os fumantes e entre os ex-fumantes apresentou mediana de, respectivamente, 11,2 maços-ano e 20,0 maços-ano. O grau de dependência demonstrou, entre os fumantes, dependência leve em 75,0\% dos indivíduos, dependência média em 17,1\% e dependência elevada em 7,9\%; entre os ex-fumantes, o grau de dependência leve, média e elevada ocorreu em 55,3\%, 29,1\% e $15,6 \%$, respectivamente 15 .

Segundo estudo de Manzano, et al. ${ }^{5}$, analisando um público diferente do presente trabalho, metade dos indivíduos em ambiente universitário consumiam menos de 10 cigarros por dia e tinham o índice de dependência a nicotina muito baixo. As pessoas que apresentaram um alto grau de dependência à nicotina consumiam em media 30 cigarros ao dia e possuíam sintomas como falta de ar e cansaço. Os valores espirométricos não apresentaram alterações significativas, apenas quatro indivíduos dos 30 pesquisados tiveram seus valores reduzidos na variável FEF25-75\%5. O presente trabalho demonstrou esses resultados, apresentando correlação positiva e significativa entre carga tabágica, dependência à nicotina e à dispneia, associando ainda dispneia aos parâmetros espirométricos obstrutivos como VEF1, VEF1/CVF e, principalmente, FEF25-75, confirmando que a alteração desta última variável está relacionada à obstrução das vias aéreas de pequeno calibre, sendo a primeira disfunção detectada em distúrbios ventilatórios obstrutivos.

Conforme estudo de Cardoso et al $^{12}$ sobre fatores relacionados ao tabagismo e a seu abandono em um estudo prospectivo mostrou que a maioria dos entrevistados teve dependência leve; isso se deve a que o estudo abordou pessoas com variados níveis de tabagismo ${ }^{12}$. Em estudo de Halty et $a^{14}$. sobre a análise da utilização do Questionário de Tolerância de Fagerström evidenciou que a metade dos fumantes regulares possui elevada dependência a nicotina, conforme aumenta a idade, aumenta o grau de dependência; a maioria dos entrevistados que possui um alto grau de dependência a nicotina tem a idade entre 51 a 60 anos, e que ainda existe uma relação entre a elevada dependência à nicotina ao menor tempo em que fuma o primeiro cigarro ao dia e a maior quantidade de cigarros consumidos4. Segundo ainda um estudo de Denise et al. ${ }^{16} \mathrm{em}$ um programa de busca de doentes com DPOC realizado em algumas capitais brasileiras evidenciou que a maior parte dos indivíduos eram do sexo masculino, com uma média de 52 anos de idade, cuja metade era fumante ativo e a outra metade era formada por ex-fumantes. Muitos dos indivíduos avaliados não apresentaram alterações na funcionalidade pulmonar ${ }^{16}$.

Com relação à função pulmonar, as variáveis relacionadas à disfunção ventilatória obstrutivas (VEF1, VEF1/CVF e FEF2575) foram associadas com o tabagismo e a dispneia. A força respiratória não apresentou associação com essas variáveis. Sabidamente, o efeito do tabagismo é conhecido na função pulmonar8. No estudo de Yaksic et $\mathrm{al}^{17}$, que analisa o perfil de uma população brasileira com grave doença pulmonar obstrutiva crônica foi observado que o VEF1 médio do grupo foi $35 \pm 14 \%$ e $78,8 \%$ eram ex-tabagistas, com tempo médio de tabagismo de $38 \pm 11$ anos/maço. Nesse contexto, é evidenciada a importância da avaliação espirometrica para mostrar o grau de acometimento pulmonar do tabagismo bem como para o acompanhamento de tabagistas e ex-tabagistas ${ }^{17}$. Godoy et $\mathrm{al}^{9}$. afirmam que a avaliação espirométrica é uma ferramenta útil para o diagnóstico de DPOC ou para avaliar indivíduos em risco para a doença9. Segundo pesquisa do ministério da saúde e do instituto nacional do câncer, apenas um pequeno número de tabagistas que frequentam consultas médicas são orientados a interromper o tabagismo e também quanto aos efeitos dos componentes tóxicos do tabaco ${ }^{2,18,19}$.

No entanto, os resultados referentes à utilização da espirometria como ferramenta motivacional nos programas de controle ao tabagismo permanecem incertos ${ }^{20,21}$. De qualquer forma, há a indicação de utilizar a espirometria para rastreamento de doenças pulmonares e a personalização das estratégias para cessação tabágica.

Entre as limitações do presente trabalho, pode-se citar a pequena quantidade de UBS que participaram do programa de controle do tabagismo do SUS em Tubarão, SC e a perda amostral referente à dificuldade de contato telefônico ou falta à avaliação.

\section{CONCLUSÃO}

De forma geral, a amostra avaliada apresentou elevado índice de consumo de cigarros e dependência à nicotina. As características como gênero, renda e escolaridade estão de acordo com outros estudos relacionados a programas antitabágicos. Foi demonstrado que, quanto maior a carga tabágica, maior a disfunção ventilatória obstrutiva. A utilização do exame espirométrico com o propósito de realizar uma avaliação inicial dos fumantes admitidos em um programa antitabágico do SUS em Tubarão, pode ser uma ferramenta útil para o diagnóstico precoce da DPOC.

\section{REFERÊNCIAS}

1. Pan American Health Organization. International health: a north south debate. Washington (DC): PASB; 1992.

2. A situação do tabagismo no Brasil. Rio de Janeiro: Ministério da saúde, 2011. Disponível no link: http://www.pneumologia.med.br/pdf/situacao_tabagismo. pdf.

3. Oliveira AF, Valente JG, Leite IC. Aspectos da mortalidade atribuível ao tabaco: revisão sistemática. Rev. Saúde Pública. 2008 abr.; 42(2):335-45. doi: http:// dx.doi.org/10.1590/S0034-89102008005000001.

4. Halty LS, Huttner MD, Oliveira Neto C, Santos VA, Martins G. Análise da utilização do Questionário de Tolerância de Fagerström (QTF) como instrumento de medida da dependência nicotínica. J. Pneumologia. 2002 jul-ago; 28(4):1806. doi: http://dx.doi.org/10.1590/S0102-35862002000400002. 
5. Manzano BM, Ramos EMC, Vanderlei LCM, Ramos D. Tabagismo no ambiente universitário: grau de dependência, sintomas respiratórios e função pulmonar. Arq. Ciênc Saúde UNIPAR 2009 maio-ago;13(2):75-80.

6. Pereira CAC. Espirometria. J Pneumol 2002 out.;28(Supl 3): 1-82

7. Souza RB. Pressões respiratórias estáticas máximas. J Pneumol 2002 out.;28(Supl 3): 155-65.

8. II Consenso Brasileiro sobre Doença Pulmonar Obstrutiva Crónica - DPOC 2004. J Bras Pneumol 2004 nov.; 30 (Supl 5).

9. Godoy I, Tanni SE, Coelho LS, Martin RSS, Parenti LC, Andrade LM, et al. Programa de cessação de tabagismo como ferramenta para o diagnóstico precoce de doença pulmonar obstrutiva crônica. J. bras. pneumol 2007 mao-jun; 33(3), p. 282-6. doi: http://dx.doi.org/10.1590/S1806-37132007000300009.

10. Gomes R, Nascimento EF, Araújo FC. Por que os homens buscam menos os serviços de saúde do que as mulheres? As explicações de homens com baixa escolaridade e homens com ensino superior. Cad. Saúde Pública. 2007 jan-mar; 23(3):565-74. doi: http://dx.doi.org/10.1590/S0102-311X2007000300015.

11. Malta DC, Moura EC, Silva SA, Oliveira PPV, Costa e Silva VL. Prevalência do tabagismo em adultos residentes nas capitais dos estados e no Distrito Federal, Brasil, 2008. J Bras Pneumol. 2010 jan.-fev.;36(1):75-83. doi: http://dx.doi. org/10.1590/S1806-37132010000100013.

12. Cardoso DB, Coelho APCP, Rodrigues M, Petroianu A. Fatores relacionados ao tabagismo e ao seu abandono. Rev Med (São Paulo). 2010 abr.-jun.;89(2):76-82.

13. Caram LMO, Ferrari R, Tanni SE, Coelho LS, Godoy I, Martin RS, et al. Perfil de fumantes atendidos em serviço público para tratamento do tabagismo. J. bras. pneumol. 2009 out.; 35(10):980-5. doi: http://dx.doi.org/10.1590/S180637132009001000006 .
14. Karen SKK, Oliveira ML, Pádua AI, Vieira F, Martinez JAB. Características clínicas de fumantes atendidos em um centro de referência na cessação do tabagismo. Medicina (Ribeirão Preto). 2012;45(3):337-42

15. Silva RLF, Carmes ER, Schwartz AF, Blaszkowski DS, Cirino RHD, Ducci RDP. Cessação de tabagismo em pacientes de um hospital universitário em Curitiba. J. bras. pneumol. 2011 jul.-ago.; 37(4):480-7. doi: http://dx.doi.org/10.1590/ S1806-37132011000400010.

16. Neves DD, Campos H, Pereira FS, Santos ND, Brito VAD, Souza IFA, et al. Tabagismo e função pulmonar em programas de busca de doentes com doença pulmonar obstrutiva crônica (DPOC). Pulmão. 2005; 14(4): 294-299.

17. Yaksic MS, Tojo M, Cukier A, Stelmach R. Profile of a Brazilian population with severe chronic obstructive pulmonary disease. J. Pneumologia. 2003 marapr; 29(2):64-8. doi: http://dx.doi.org/10.1590/S0102-35862003000200004.

18. Ministério da Saúde (Brasil). Instituto Nacional de Câncer - INCA Coordenação de Prevenção e Vigilância (CONPREV). Abordagem e Tratamento do Fumante: consenso 2001. Rio de Janeiro: INCA; 2001.

19. Oliveira AF, Valente JG, Leite IC. Aspectos da mortalidade atribuível ao tabaco: revisão sistemática. Rev. Saúde Pública 2008 abr; 42(2):335-45. doi: http://dx.doi.org/10.1590/S0034-89102008005000001.

20. Wilt TJ, Niewoehner D, Kane RL, MacDonald R, Joseph AM. Spirometry as a motivational tool to improve smoking cessation rates: a systematic review of the literature. Nicotine Tob Res. 2007 Jan; 9(1):21-32. PubMed PMID: 17365733.

21. Buffels J, Degryse J, Decramer M, Heyrman J. Spirometry and smoking cessation advice in general practice: a randomised clinical trial. Respiratory Medicine. 2006 Nov;100(11):2012-7. PubMed PMID: 16580189. 